\title{
ESTUDO E CULTIVO DE PLANTAS MEDICINAIS COM ATIVIDADE REPELENTE DE INSETOS: UMA PROPOSTA DE EDUCAÇÃO EM SAÚDE
}

Investigación y cultivo de plantas medicinales con actividade repelente de insectos: una propuesta de educación en salud

Study and cultivation of medicinal plants with insect repellent activity: a proposal of health education

\section{Juliana Carvalho Ribeiro' ${ }^{1}$, Yula de Lima Merola²}

\section{RESUMO}

As doenças transmitidas pelo inseto Aedes aegypt são consideradas um desafio para a saúde pública. No intuito de desenvolver ações de educação em saúde para minimizar a presença desse vetor, este projeto promoveu um estudo e uma ação social para a distribuição de mudas de plantas medicinais com atividade repelente de insetos na cidade de Poços de Caldas. A proposta foi um sucesso e foi uma ferramenta de grande valia, fortalecendo o elo entre a comunidade acadêmica, a Fundação Jardim Botânico de Poços de Caldas e a população atendida.

Palavras-chave: Aedes aegypt; Plantas repelentes de inseto; Educação em saúde.

\footnotetext{
${ }^{1}$ Doutora em Ciências pela Faculdade de Ciências Farmacêuticas de Ribeirão Preto (USP), Professora adjunta do curso de graduação em Farmácia e Enfermagem no Centro Universitário das Faculdades Associadas de Ensino (UNIFAE).2 Doutora em Ciências da Saúde pela Faculdade de Ciências Médicas da ${ }^{2}$ ${ }^{2}$ Universidade de Campinas (UNICAMP), Professora da Faculdade de Pitagoras.
} 


\section{RESUMEN}

Las enfermedades transmitidas por el insecto Aedes aegypt son consideradas un desafío para la salud pública. A fin de desarrollar acciones de educación en salud para minimizar la presencia de este vector, este proyecto promovió una investigación y una acción social para la distribuciónde plantas medicinales con actividad repelente de insectos en la ciudad de Poços de Caldas. La propuesta obtuvo éxito y fue una herramienta de granvalor, fortaleciendo ellazo entre la comunidad académica, la Fundación Jardín Botánico de Poços de Caldas e lapoblación atendida.

Palabras Clave: Aedes aegypt; Plantas repelentes de insecto; Educación en salud.

\section{ABSTRACT}

The diseases transmitted by the insect Aedes aegypt are considered a challenge for public health. In order to develop health education actions to minimize the presence of this vector, this project promoted a study and a social action for the distribution of medicinal plant seedlings with insect repellent activity in the city of Poços de Caldas. The proposal was a success and was a valuable tool, strengthening the link between the academic community, the Jardim Botânico Foundation of Poços de Caldas and the attended population.

Keywords: Aedes aegypt; Insect repellent plants; Health education 


\section{EM QUE CONSISTE A PRÁTICA A SER RELATADA}

O termo doenças tropicais é usado para conceituar as doenças mais presentes na região dos trópicos e que estão intimamente relacionadas com as variáveis climáticas e as condições políticas, econômicas e socioambientais. Nesse âmbito, a dengue é considerada uma doença tropical, pois as condições climáticas dessas regiões facilitam a manutenção do mosquito responsável pela sua transmissão (SILVA, 2015).

Atualmente, a dengue é um grave problema de saúde pública nos países em desenvolvimento (ARAÚJO et al., 2008), e há anos tem-se realizado estudos para controlar ou mesmo minimizar o problema, porém ainda continua a ser uma situação preocupante a nível mundial.

A doença tem como agente etiológico um vírus do gênero Flavivírus pertencente à família Flaviviridae, transmitida no Brasil pelo vetor mosquito Aedes aegypti (SILVA, 2015). Considera-se que sua distribuição geográfica é mundial, envolvendo países tropicais e subtropicais (BRAGA; VALLE, 2007).

Apesar de inúmeros esforços para a erradicação da dengue, nota-se que sua distribuição geográfica tem sido considerada desigual entre os países, e dentro dos próprios países. Algumas explicações para esta distribuição desigual estão relacionadas com determinantes sociais. Alguns estudos relatam que fatores como a presença de canalização de água interna, de coleta de lixo, de ligação sanitária, número de moradores no domicílio, renda da família e grau de escolaridade podem influenciar diretamente na maior incidência de criadouros do mosquito Aedes aegypt (ARAÚJO et al., 2008). O mosquito transmissor se reproduz em locais onde há água parada como, por exemplo, pneus, depósitos de ferros velhos descobertos, latas, garrafas, plásticos abandonados e terrenos baldios. A eliminação do mosquito acontece por meio da eliminação dos criadouros, uso de inseticida, principalmente durante a época de transmissão, e apoio da população (SILVA, 2015).

Ainda existem diversas questões a serem esclarecidas sobre a doença, desde a eliminação dos locais onde os mosquitos se reproduzem até o combate à cadeia de transmissão da doença com o desenvolvimento de políticas nacionais de controle da mesma. Pelo fato de grande parte dos criadouros infestados ou potenciais se encontrarem no interior dos domicílios, as atividades educativas têm cada vez mais responsabilidades, tanto no engajamento da população na eliminação dos criadouros, como no esclarecimento sobre a dengue e sua etiologia (SALES, 2008).

Para o combate ao mosquito são comumente utilizados os inseticidas sintéticos. Entretanto, o consumo intensivo desses produtos tem acarretado em resistentes nas populações dos mosquitos (LUNA et al., 2004). Dessa forma e devido à grande diversidade botânica do Brasil, tem-se estuda- 
do e aplicado inúmeras plantas detentoras de atividade inseticida, o que corrobora para uma maior investigação e introdução destas como opção no controle integrado de pragas (ROEL et al., 2000).

Neste cenário, Rangel(2008) descreve a importância da educação, comunicação e mobilização na perspectiva de controle da dengue e que as práticas de comunicação e educação realizadas para o controle da dengue não se diferenciam daquelas hegemônicas no campo da Saúde Pública no nosso país. Ainda, ressalta que é importante relativizar o poder dessas práticas em produzir ou induzir mudanças de comportamentos e atitudes, especialmente em contextos tão adversos à proteção e promoção da saúde. A comunicação, educação e mobilização social são campos de ação fundamentais para o bom desempenho de programas de prevenção e promoção da saúde (RANGEL, 2008).

O Ministério da saúde define a educação em saúde como:

\footnotetext{
"Processo educativo de construção de conhecimentos em saúde que visa à apropriação temática pela população [...]. Conjunto de práticas do setor que contribui para aumentar a autonomia das pessoas no seu cuidado e no debate com os profissionais e os gestores a fim de alcançar uma atenção de saúde de acordo com suas necessidades" (FALKENBERG et al., 2014).
}

As práticas de educação em saúde envolvem três segmentos prioritários, envolvendo os profissionais de saúde para que valorizem a prevenção e a promoção tanto quanto as práticas curativas; os gestores para que apoiem esses profissionais; e a população que necessita construir seus conhecimentos e aumentar sua autonomia nos cuidados, individual e coletivamente. Embora a definição do Ministério da Saúde apresente elementos que pressupõem essa interação entre os três segmentos das estratégias utilizadas para o desenvolvimento desse processo, ainda existe grande distância entre retórica e prática (FALKENBERG et al., 2014).

Educação para a saúde também é outro termo usual ainda hoje nos serviços de saúde. Aqui se supõe uma concepção mais verticalizada dos métodos e práticas educativas, que remete ao que Freire (1987) chamou de educação bancária. Analisando este conceito, é como se os profissionais de saúde devessem ensinar uma população o que precisaria ser feito para a mudança de hábitos de vida, a fim de melhorar a saúde individual e coletiva (FALKENBERG et al., 2014).

Em função de diversas pesquisas, sabe-se que além da dengue, o Aedes aegypti é transmissor 
da febre chikungunya e febre amarela. Assim, ações que intensificam a educação em saúde para a eliminação do vetor são importantes, influenciando diretamente na diminuição dos casos dessas doenças. No intuito de preparar os profissionais em saúde para serem os protagonistas de práticas de educação em saúde é indispensável que na sua formação acadêmica hajam propostas de projetos evidenciando esta prática.

\section{CONTEXTO EM QUE OCORRE A AÇÃO}

O objetivo deste estudo foi propor a execução de um projeto de campo, na forma de estágio, envolvendo alunos em estudos sobre plantas medicinais com atividade repelente de Aedes aegypt, produção de mudas e ação de distribuição dessas mudas para a população da cidade de Poços de Caldas, Minas Gerais, em uma ação social voltada para a conscientização sobre a erradicação do mosquito Aedes aegypt.

\section{PARTICIPANTES/INTEGRANTES DA AÇÃO RELATADA}

Participaram do projeto quinze alunos dos cursos de Farmácia e Engenharia Ambiental da Faculdade Pitágoras de Poços de Caldas, Minas Gerais, classificados em um processo seletivo, no período de março a junho de 2016.

\section{METODOLOGIA}

O projeto foi dividido em duas partes, sendo uma parte teórica e uma parte prática. A parte teórica abordou pesquisa bibliográfica, elaboração e apresentação do projeto de pesquisa para a professora responsável pelo estágio e também apresentação do relatório final para a coordenação do curso de Farmácia da Faculdade Pitágoras e para a Fundação Jardim Botânico de Poços de Caldas. A revisão bibliográfica foi realizada em grupos de três alunos e norteou as demais ações. A busca dos trabalhos foi realizada nas bases de dados Pubmed, Google Acadêmico e Scielo. A seleção foi realizada por critérios de inclusão, sendo estes: artigos originais sobre dengue e plantas medicinais com atividade repelente de insetos publicados em inglês ou português de 1998 a 2015; trabalhos de conclusão de curso; e trabalhos apresentados em congresso. Para procurar plantas ao alcance da população brasileira, trabalhos realizados em outros países foram excluídos. As palavras-chave utilizadas na identificação dos artigos foram: "dengue", "plantas medicinais e dengue", "Aedes aegypti", "repelentes de insetos" e suas traduções para língua inglesa. 
A parte prática foi subdividida no desenvolvimento de mudas de plantas medicinais com propriedades repelentes do Aedes aegypt, ação social para a distribuição destas mudas e visita técnica a um centro de pesquisa em plantas medicinais. Após uma revisão bibliográfica inicial, houve a seleção e definição da quantidade das mudas a serem desenvolvidas. As mudas foram produzidas utilizando a estrutura da Fundação Jardim Botânico de Poços de Caldas, a partir de material propagativo identificado e catalogado pelo herbário local. Ao longo de dois meses, os participantes frequentaram semanalmente as dependências da Fundação Jardim Botânico de Poços de Caldas, passando por treinamento de técnicas agronômicas, executando o plantio e acompanhamento do desenvolvimento de 590 mudas de alecrim, citronela e manjericão. A ação social foi realizada também em parceria com a Fundação Jardim Botânico de Poços de Caldas, quando foi montada uma estrutura em local definido, onde foi feita a distribuição das mudas produzidas. Para a distribuição, os alunos passaram por treinamento, realizando a comunicação verbal e por panfletos.

Ao longo desse período, a equipe também participou de uma visita técnica à Coleção de Plantas Medicinais e Aromáticas da Unicamp (CPMA), veiculada ao Centro Pluridisciplinar de Pesquisas Químicas, Biológicas e Agrícolas (CPQBA), situado na cidade de Paulínia, São Paulo.

\section{RESULTADOS ALCANÇADOS}

A pesquisa bibliográfica realizada pela equipe evidenciou que plantas contendo compostos fitoquímicos que atuam como repelentes de insetos são alternativas promissoras para o auxílio no combate do Aedes aegypti. Estudos descrevem a atividade repelente de plantas medicinais, entre elas a citronela (Cymbopogon winterianus), o alecrim (Rosmarinus officinalis), manjericão (Ocimum basilicum), urundeuva (Myracrodruon urundeuva), moringa (Moringa oleifera), crotalária (Crotalaria verrucosa), dentre outras plantas (LINDSAY, 1996; SANTOS, 2008; MURUGAN, et al., 2016; VELOSO et al., 2015). Puccini et al. (2015) fez uma análise comparativa entre as bases de dados Pubmed, Scielo e Google Acadêmico com o foco na temática Educação Médica. A comunidade biomédica latino-americana tem o Pubmed e o Scielo como as melhores bases de dados para pesquisa de artigos científicos. Entretanto, outras áreas de conhecimento têm utilizado outras fontes de busca, sendo a principal o Google Acadêmico. Este estudo mostrou que o Google Acadêmico mostrou ser mais eficiente, tanto qualitativamente quanto quantitativamente, em resgatar artigos científicos no tema escolhido em Educação Médica. Assim, conclui-se que a pesquisa bibliográfica norteadora desta atividade de educação em saúde oferece qualidade nos dados encontrados.

Na Fundação Jardim Botânico de Poços de Caldas, os alunos tiveram noções teóricas e práticas sobre plantio e cultivo de plantas medicinais visando os aspectos agronômicos, o que foi muito 
interessante, pois o aluno do curso de farmácia tem como foco conceitos voltados à ação farmacológica, dosagem e desenvolvimento de formas farmacêuticas fitoterápicas e o curso de engenharia ambiental não aborda especificamente o cultivo de plantas medicinais.

$\mathrm{Na}$ ação social, os alunos distribuíram as mudas desenvolvidas e exerceram a atividade de educação em saúde, utilizando as plantas medicinais como ferramenta no combate ao vetor de doenças diretamente relacionadas aos atuais problemas de saúde pública. Usando a comunicação verbal com vocabulário cotidiano e também panfletos informativos, constatamos que foi um evento de sucesso, com ampla participação dos alunos envolvidos e grande aceitação pela comunidade local.

Os alunos se envolveram muito com o tema e a aceitação e o retorno foram visíveis, distribuindo as 590 mudas desenvolvidas. Freitas, Rezende Filho (2010) realizaram uma revisão bibliográfica sobre modelos de comunicação e uso de impressos na educação em saúde. Neste estudo, relata-se que muitos panfletos usados em ações de educação em saúde se aproximam muito da linguagem biomédica, outros fazem uma tradução da linguagem científica para a linguagem popular. Ainda, nos artigos pesquisados pode-se encontrar um conflito entre a perspectiva dos seus autores e a dos produtores dos impressos. No caso desta ação social, os panfletos foram de fácil interpretação, com letras grandes e ilustrações, facilitando a compreensão da comunidade.

Na figura 1 é possível observar o grupo durante a ação de educação em saúde para a entrega e orientação sobre o uso de plantas medicinais com atividade repelente de insetos.

Figura 1: Ação de educação em saúde realizada na praça central de Poços de Caldas, MG.
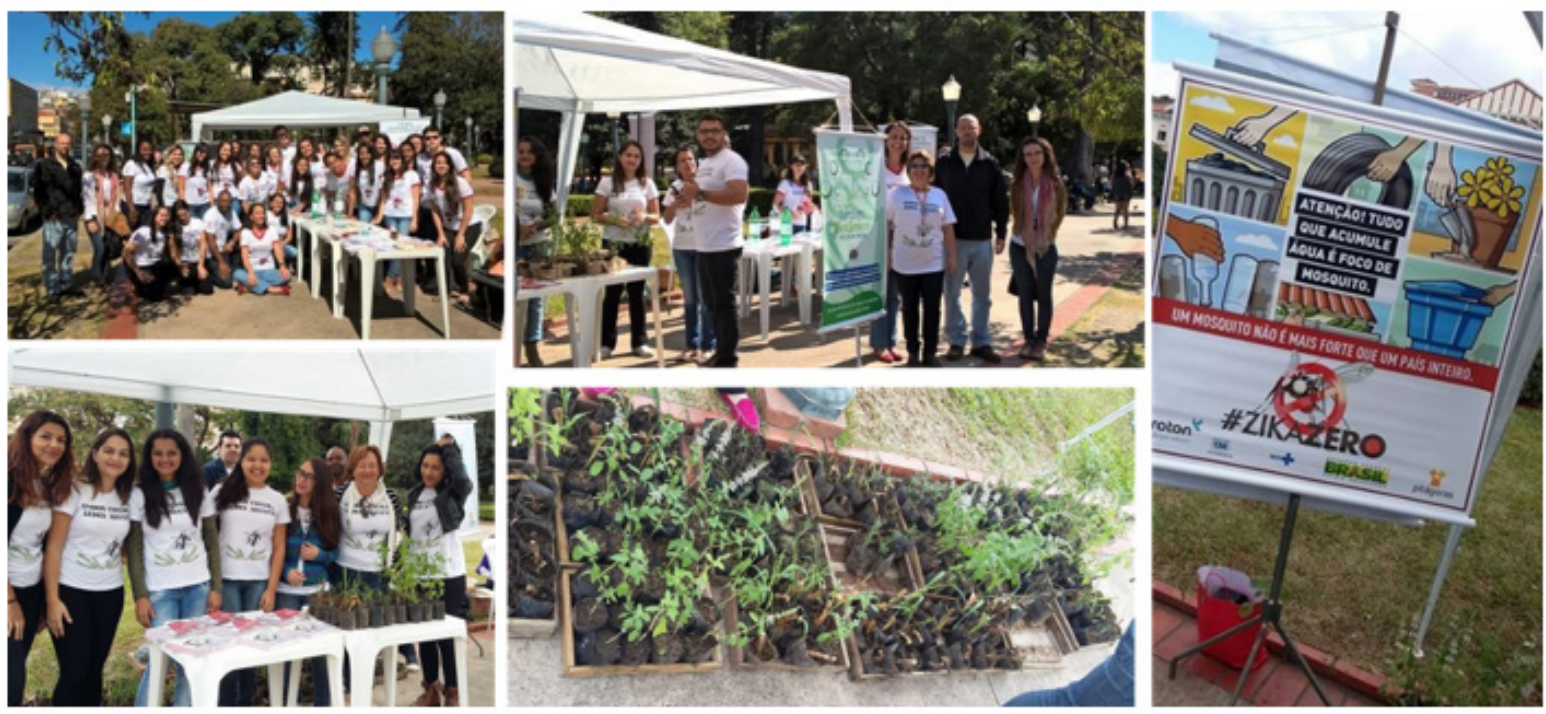

Fonte: Arquivos dos autores 
Na visita à Coleção de Plantas Medicinais e Aromáticas da Unicamp (CPMA), os alunos assistiram a uma palestra sobre a importância dos estudos de fitotecnia no desenvolvimento de medicamentos fitoterápicos, visitaram os laboratórios, a parte de cultivo e desenvolvimento de mudas de plantas medicinais e também conheceram os exemplares da coleção de plantas medicinais disponíveis para as pesquisas (Figura 2).

Figura 2: Visita técnica à Coleção de Plantas Medicinais e Aromáticas da Unicamp (CPMA), vinculada ao Centro Pluridisciplinar de Pesquisas Químicas, Biológicas e Agrícolas (CPQBA), situado na cidade de Paulínia, SP.

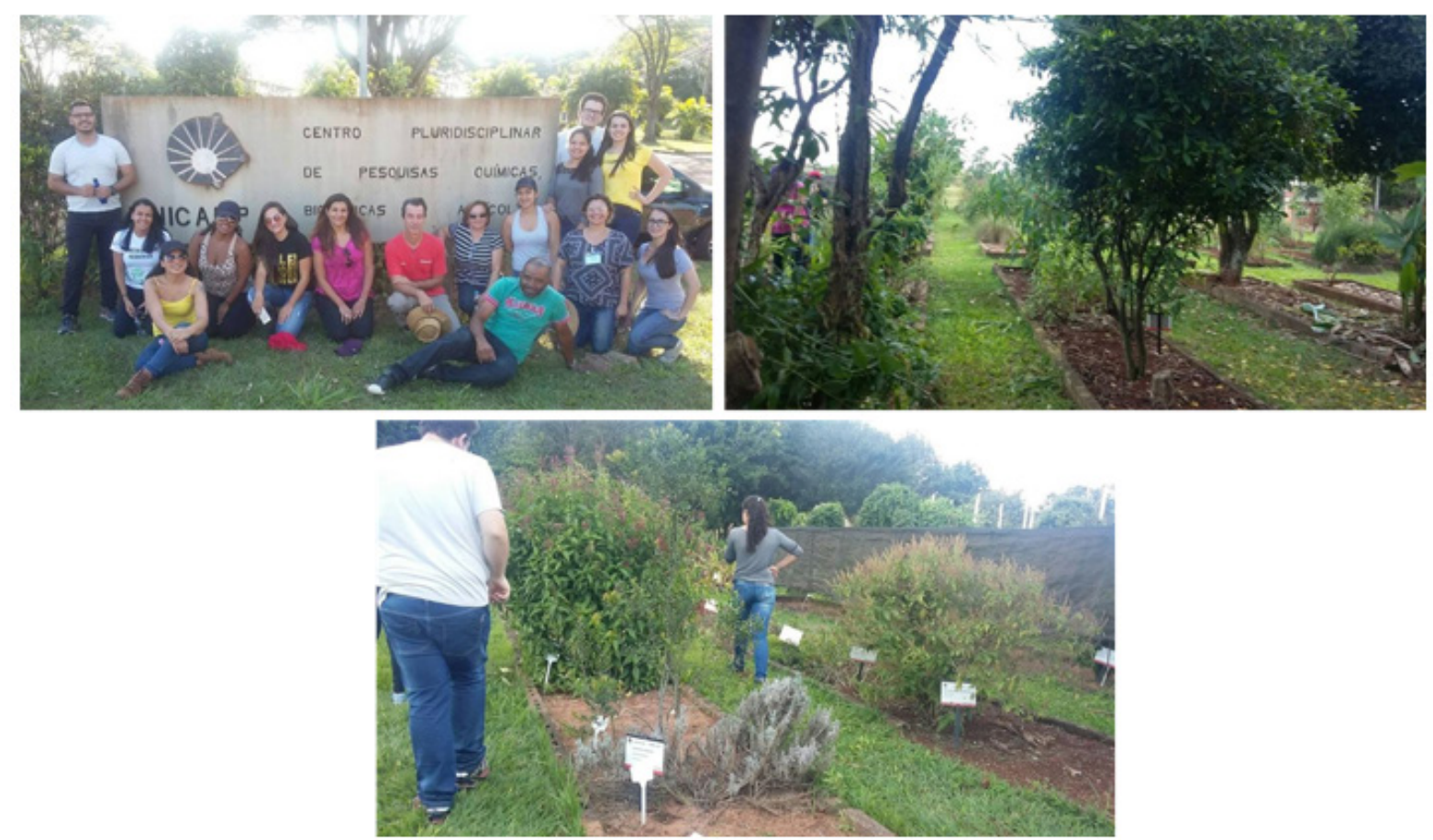

Fonte: Arquivos dos autores

Essa visita técnica foi de grande valia, pois a riqueza de detalhes vivenciados relacionados ao cultivo e pesquisas de marcadores químicos e farmacológicos foi uma nova área de conhecimento.

\section{O QUE SE APRENDEU COM A EXPERIÊNCIA}

A preparação de um profissional atuante em educação em saúde envolve estratégias de ensino diversificadas. O projeto foi executado e concluído no tempo preconizado. Os alunos participaram do cultivo de mudas de plantas medicinais, organizaram uma ação social que teve grande aceitação da população e se envolveram no estudo de plantas medicinais com atividade repelente de insetos. Ainda, tiveram a oportunidade de conhecer um grande centro de pesquisa em plantas medicinais 
que é referência no país. É importante ressaltar que a parceria com a Fundação Jardim Botânico foi fundamental para o sucesso da implantação do projeto, fortalecendo o elo entre a comunidade acadêmica e o serviço oferecido por este centro que é referência regional em educação ambiental. Infelizmente, em função de mudanças na vida profissional dos professores envolvidos e também mudanças a nível administrativo municipal, não foi possível dar continuidade ao projeto, mas é uma ideia que deve ser compartilhada e fonte de informações para estudos futuros.

\section{RELAÇÃO DA PRÁTICA COM OS CONCEITOS DE EXTENSÃO}

Esta foi uma oportunidade de observar que plantas medicinais são um assunto multidisciplinar, com parâmetros e conceitos bem definidos e estruturados para se obter exemplares de qualidade, contendo os marcadores químicos e farmacológicos em concentrações adequadas para serem usados para fins terapêuticos. Santa Anna (2019) avaliou as potencialidades das visitas técnicas na docência universitária e constatou que as visitas técnicas funcionam como instrumentos pedagógicos que provocam a motivação e engajamento dos participantes com o aprendizado, como também promovem a identificação e a visibilidade das práticas de catalogação em acervos bibliográficos. Em síntese, as visitas muito contribuem para a fusão entre teoria e prática; entre sala de aula e mercado de trabalho.

Ações educativas para prevenção e controle da dengue apontam limites e dificuldades. Neste caso, não foi feito um estudo minucioso de medição das dificuldades e limitações na execução deste projeto. Porém, ressalta-se que este foi um trabalho pioneiro na cidade e não foram encontrados trabalhos semelhantes na literatura para fins de comparação de resultados.

\section{AGRADECIMENTOS}

Fundação Jardim Botânico de Poços de Caldas e Coleção de Plantas Medicinais e Aromáticas da Unicamp (CPMA), veiculada ao Centro Pluridisciplinar de Pesquisas Químicas, Biológicas e Agrícolas (CPQBA).

\section{REFERÊNCIAS}

ARAÚJO, José Raimundo de; FERREIRA, Efigênia Ferreira; ABREU, Mauro Henrique Nogueira Guimarães de. Revisão sistemática sobre estudos de espacialização da dengue no Brasil. Revista brasileira de epidemiologia, v. 11, p. 696-708, 2008. Disponível em: https://doi.org/10.1590/ S1415-790X2008000400016. Acesso em: 27 fev. 2021. 
BRAGA, Ima Aparecida; VALLE, Denise. Aedes aegypti: histórico do controle no Brasil. Epidemiologia e serviços de saúde, v. 16, n. 2, p. 113-118, 2007. Disponível em:http://dx.doi.org/10.5123/ S1679-49742007000200006. Acesso em: 27 fev. 2021.

FALKENBERG, Mirian Benites et al. Educação em saúde e educação na saúde: conceitos e implicações para a saúde coletiva. Ciência \& Saúde Coletiva, v. 19, p. 847-852, 2014. Disponível em:https://doi.org/10.1590/1413-81232014193.01572013. Acesso em: 27 fev. 2021.

FREIRE, Paulo. Pedagogia do oprimido. 17ª Edição. São Paulo: Paz e Terra, 1987.

FREITAS, Fernanda Valéria de; REZENDE FILHO, Luiz Augusto. Modelos de comunicação e uso de impressos na educação em saúde: uma pesquisa bibliográfica. Interface-Comunicação, Saúde, Educação, v. 15, p. 243-256, 2010. Disponível em: https://doi.org/10.1590/S141432832010005000044. Acesso em: 27 fev. 2021.

LINDSAY, Robbin et al. Evaluation of the efficacy of 3 Vo citronella candles and 5Vo citronella incense for protection against field populations of Aedes mosquitoes. Journal of the American Mosquito Control Association, v. 12, n. 2, p. 293-294, 1996. Disponível em:https://pubmed.ncbi. nlm.nih.gov/8827606/. Acesso em: 27 fev. 2021.

LUNA, Jonny Edward Duque et al. Susceptibilidade de Aedes aegypti aos inseticidas temephos e cipermetrina, Brasil. Revista de Saúde Pública, n. 38, p. 842-843, 2004.

MURUGAN, Kadarkarai et al. Rapid biosynthesis of silver nanoparticles using Crotalaria verrucosa leaves against the dengue vector Aedes aegypti: what happens around? An analysis of dragonfly predatory behaviour after exposure at ultra-low doses. Natural Product Research, v. 30, n. 7, p. 826-833, 2016. Disponível em:https://doi.org/10.1080/14786419.2015.1074230. Acesso em: 27 fev. 2021.

PUCCINI, Lucas Rebelo Silva et al. Comparativo entre as bases de dados PubMed, SciELO e Google Acadêmico com o foco na temática Educação Médica. Cadernos UniFOA, v. 10, n. 28, p. 7582, 2015.Disponível em: https://core.ac.uk/download/pdf/287324521.pdf. Acesso em: 27 fev. 2021. 
RANGEL, Maria Ligia. Dengue: educação, comunicação e mobilização na perspectiva do controle-propostas inovadoras. Interface-Comunicação, Saúde, Educação, v. 12, p. 433-441, 2008. Disponível em:https://www.scielosp.org/article/icse/2008.v12n25/433-441/pt/. Acesso em: 27 fev. 2021.

ROEL, Antonia Railda et al. Atividade tóxica de extratos orgânicos de Trichilia pallida Swartz (Meliaceae) sobre Spodoptera frugiperda (J.E. Smith). Anais da Sociedade Entomológica do Brasil, v.29, p.799-808, 2000.

SANTA ANNA, Jorge. Potencialidades das visitas técnicas na docência universitária: aplicações nas disciplinas de representação da informação. Revista Internacional de Educação Superior, v. 5, p. e019005-e019005, 2019. Disponível em: https://periodicos.sbu.unicamp.br/ojs/index.php/ riesup/article/view/8653104. Acesso em: 27 fev. 2021.

SALES, Fátima Maria de Sousa. Ações de educação em saúde para prevenção e controle da dengue: um estudo em Icaraí, Caucaia, Ceará. Ciência \& Saúde Coletiva, v. 13, n. 1, p. 175-184, 2008. Disponível em: https://www.scielo.br/scielo.php?pid=S1413-81232008000100022\&script=sci_arttext. Acesso em: 27 fev. 2021.

SANTOS, Nataly Diniz de Lima. Efeito da lectina de sementes de Moringa oleifera (WSMoL) sobre o desenvolvimento larval e mortalidade de Aedes aegypti. 2008. Dissertação de Mestrado. Universidade Federal de Pernambuco. Disponível em:https://attena.ufpe.br/bitstream/123456789/1549/2/ndls.pdf. Acesso em: 27 fev. 2021.

SILVA, Ivanise Brito da; MALLMANN, Danielli Gavião; DE VASCONCELOS, Eliane Maria Ribeiro. Estratégias de combate à dengue através da educação em saúde: uma revisão integrativa. Saúde (Santa Maria), v. 41, n. 2, p. 27-34, 2015. Disponível em: https://periodicos.ufsm.br/index. php/revistasaude/article/view/10955. Acesso em: 27 fev. 2021.

VELOSO, Ronice Alves et al. Óleos essenciais de manjericão e capim citronela no controle de larvas de Aedes aegypti. Revista Verde de Agroecologia e Desenvolvimento Sustentável, v. 10, n. 2, p. 101-105, 2015. Disponível em: https://editoraverde.org/gvaa.com.br/revista/index.php/RVADS/ article/view/3322. Acesso em: 27 fev. 2021. 